\title{
An Urban Case of Canine Visceral Leishmaniasis in the Municipality of Pereira, Colombia
}

\author{
Luz Victoria González-Colonia ${ }^{1}$, D. Katterine Bonilla-Aldana ${ }^{2}$, María Paulina Álvarez-Serrano ${ }^{3}$, Laura Y. \\ Granados-Hincapie $^{2}$, L. Camila Pinilla-Ticora ${ }^{2}$, and Alfonso J. Rodríguez-Morales ${ }^{2,4} *$ \\ ${ }^{I}$ San Lucas Centro Veterinario y Diagnostico, Pereira, Risaralda, Colombia \\ ${ }^{2}$ Semillero de Investigación en Zoonosis (SIZOO), Grupo de Investigación BIOECOS, Fundación Universitaria Autónoma de Las Américas, Sede \\ Pereira, Pereira, Risaralda, Colombia \\ ${ }^{3}$ Cimev Hospital Veterinario, Armenia, Quindio, Colombia \\ ${ }^{4}$ Grupo de Investigacion Biomedicina, Faculty of Medicine, Fundacion Universitaria Autonoma de Las Americas, Pereira, Risaralda, Colombia \\ *Corresponding author's Email: alfonso.rodriguez@uam.edu.co ; (DORCiD: https://orcid.org/0000-0001-9773-2192
}

\begin{abstract}
American visceral leishmaniasis (VL) is caused by Leishmania infantum/chagasi, transmitted by the sandflies Lutzomyia longipalpis and Lu. evansi. Dogs are the main reservoir and source for zoonotic infections in humans. Therefore, it is of utmost importance to diagnose such diseases in domestic animals to maintain public health. In 2019, the authors of the present study observed intracellular amastigotes in Giemsa-stained bone marrow smear using a real-time qPCR (parasite load was 484,336 DNA copies/mL), a canine visceral leishmaniasis (CVL) case caused by $L$. infantum/chagasi, in a 22-month-old male, English bulldog from Pereira, Colombia, a municipality not previously considered endemic for CVL nor human VL. Therefore, further field studies are necessary to determine if there is a low-grade circulation of this parasite among dogs and other animal reservoirs in the area, also entomological surveys are of utmost importance, as well as highlighting the clinical suspicion of this disease in domestic animals and humans.
\end{abstract}

Keywords: Colombia, Dog, Leishmaniasis, Non-endemic area, Visceral, Zoonotic

\section{INTRODUCTION}

American visceral leishmaniasis (VL) is a tropical and subtropical vector-borne disease caused by the protozoa species Leishmania infantum/chagasi, and transmitted by Lutzomyia longipalpis and Lu. evansi phlebotomine sandflies species (Dantas-Torres et al., 2019; Arbelaez et al., 2020). Dogs are its main animal host reservoir, especially in urban areas, and they may undergo this clinical disease with severe compromise and even fatal outcomes. Moreover, other domestic animals, such as cats, may be infected and become reservoirs of this parasitic disease. Canine VL (CVL) has a highly critical position in transmission cycles, especially in urban areas, with the presence of the vectors and suitable ecoepidemiological conditions, including areas prone to susceptible climatic conditions. This issue may pose an increased risk for human cases and become a public health threat, as zoonotic transmission may occur, and this disease, especially in the case of children may lead to fatal outcomes (Dantas-Torres et al., 2019; Arbelaez et al., 2020).

In Colombia, South America, human and canine VL has been historically restricted to two transmission areas (Figure 1), one in three departments of the north Caribbean coast region (hot humid area with suitable conditions for the vector), and another in three departments of the central-south part of the middle Magdalena River Valley (Figure 1), also with confirmation of the vector presence in these zones (Arbelaez et al., 2020). Recent reports have caused concern about the occurrence of CVL in new areas of the country, previously not considered endemic, with no history of case reports of VL nor in humans or animals. A case of a 2-year-old male dog in Cali, Valle del Cauca (Figure 1), diagnosed with CVL by PCR, a region where VL has not been informed before was described in 2019 (Arbelaez et al., 2020). Bordering with Valle del Cauca and Tolima department, a previously known endemic area, is located Risaralda Department (Figure 1). The CVL case in a dog from Pereira municipality, Risaralda, Colombia was presented by the authors of the current study.

\section{Case report}

A 22-month-old castrated male English bulldog was hospitalized in a private veterinary center in the city of Pereira municipality, Risaralda Department, Colombia (Figure 1), on September 13, 2019, with a 2-month history of lack of appetite, general weakness, progressive loss of weight, and high body temperature $\left(38.9-39.6^{\circ} \mathrm{C}\right)$. Physical examination revealed that the physiological constants (heart rate, respiratory rate, or pulse) were in the normal ranges. No alterations 
were found at lymph nodes, skin, and eyes. Blood laboratory assessments using a hematology analyzer Mindray BC2800 Vet ${ }^{\circledR}$ (Guangdong Sheng, China) showed pancytopenia, with normocytic normochromic anemia (reaching 6.2 $\mathrm{g} / \mathrm{dL}$, normal range 12-18), leukopenia (reaching 4,800 cells $/ \mathrm{mm}^{3}$, normal range 6,000-17,000), thrombocytopenia (reaching 40 cells $/ \mu \mathrm{L}$, normal value $>200$ ), and lymphopenia (reaching 804 cells $/ \mathrm{mm}^{3}$, normal range 1,500-7,000). A significantly increased alanine aminotransferase (ALT) levels were also observed (213.6 U/L, normal range 4-90) and slightly on aspartate aminotransferase (AST) (47.6 U/L, normal range 16-43). Albumin, globulin, BUN, and creatinine were not analyzed. Biochemistry tests were performed with a chemistry analyzer Mindray BA 88 A®) (Guangdong Sheng, China).

A hip X-ray suggested a dysplasia at the coxo-femoral joint. An abdominal ultrasound, using a Mindray Ultrasound Z5® (Guangdong Sheng, China) showed spleen benign neoplasia, based on the finding of a heterogeneous echogenic pattern with diffuse rounded hypoechoic foci in its parenchyma, and a capsule irregular with some protuberances, typical vascular pattern, and thickness $(21.3 \mathrm{~mm})$. No alterations were found in the kidneys. Then, a bone marrow aspirate was performed with confirmation after the observation of intracellular amastigotes in Giemsa-stained bone marrow smear, observed using a light microscope (Scientific $®$, Maharashtra 400076, India) (Figure 2). A real-time qPCR using a TaqMan® probe (ThermoFisher, Foster City, CA, USA) with the bone marrow aspirate sample confirmed the diagnosis of VL due to Le. infantum. The parasite load was 484,336 DNA copies/mL (cycle threshold 19.62).

Notification of a case of CVL in a non-endemic area, which was performed when diagnosed, requires information about the places where the animal was before the diagnosis. The owner referred to two sites in the rural area of Risaralda, rural Pereira and Viterbo, rural area of Caldas, both non-endemic for VL (Figure 1). Following public health recommendations, the animal was euthanatized with the owner's authorization.
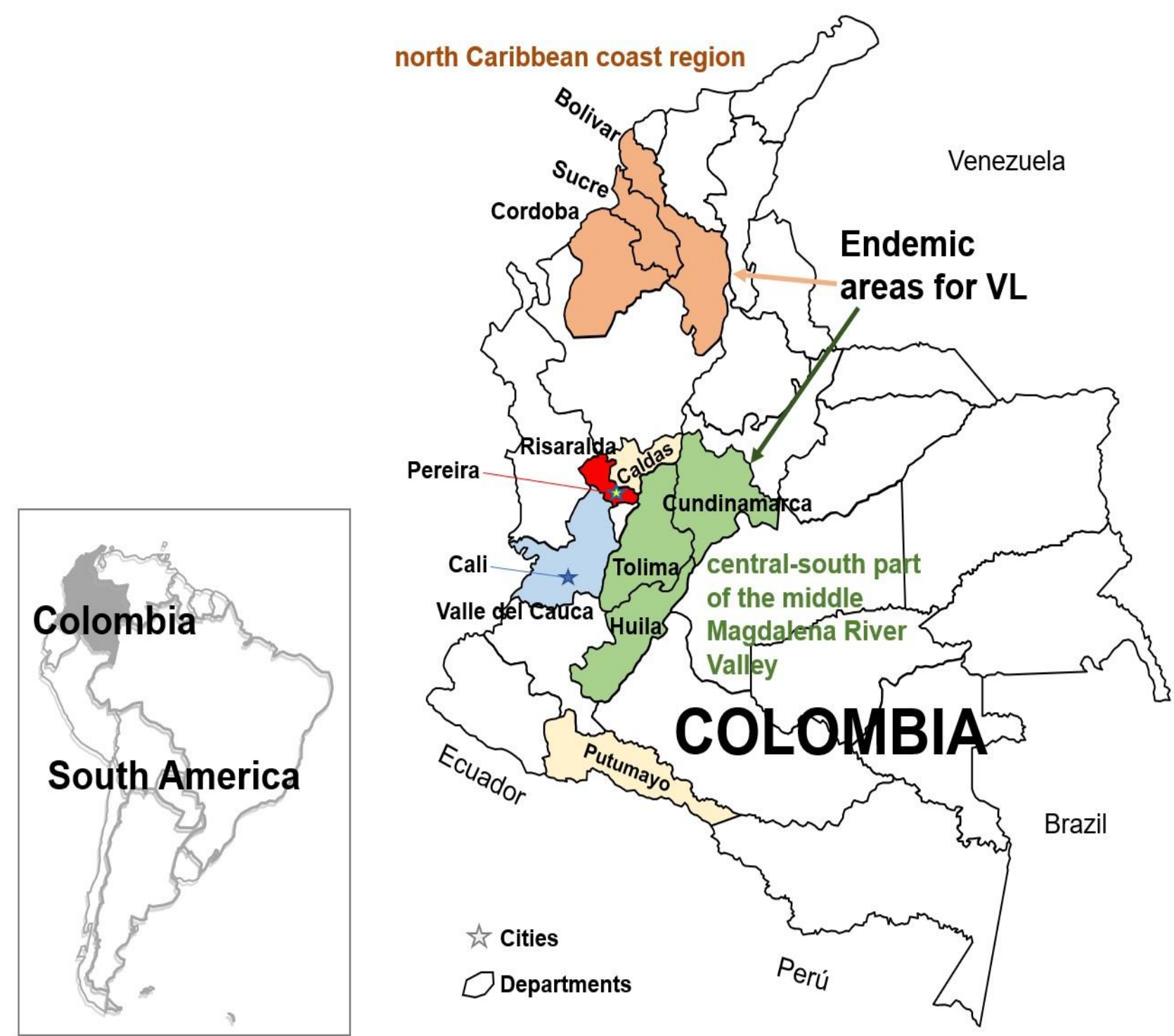

Figure 1. Relative map of Colombia showing the endemic areas for visceral leishmaniasis (VL), and the departments of Valle del Cauca, Cauca, Putumayo, and Risaralda, not considered endemic, where those previous and the current cases have been reported. 


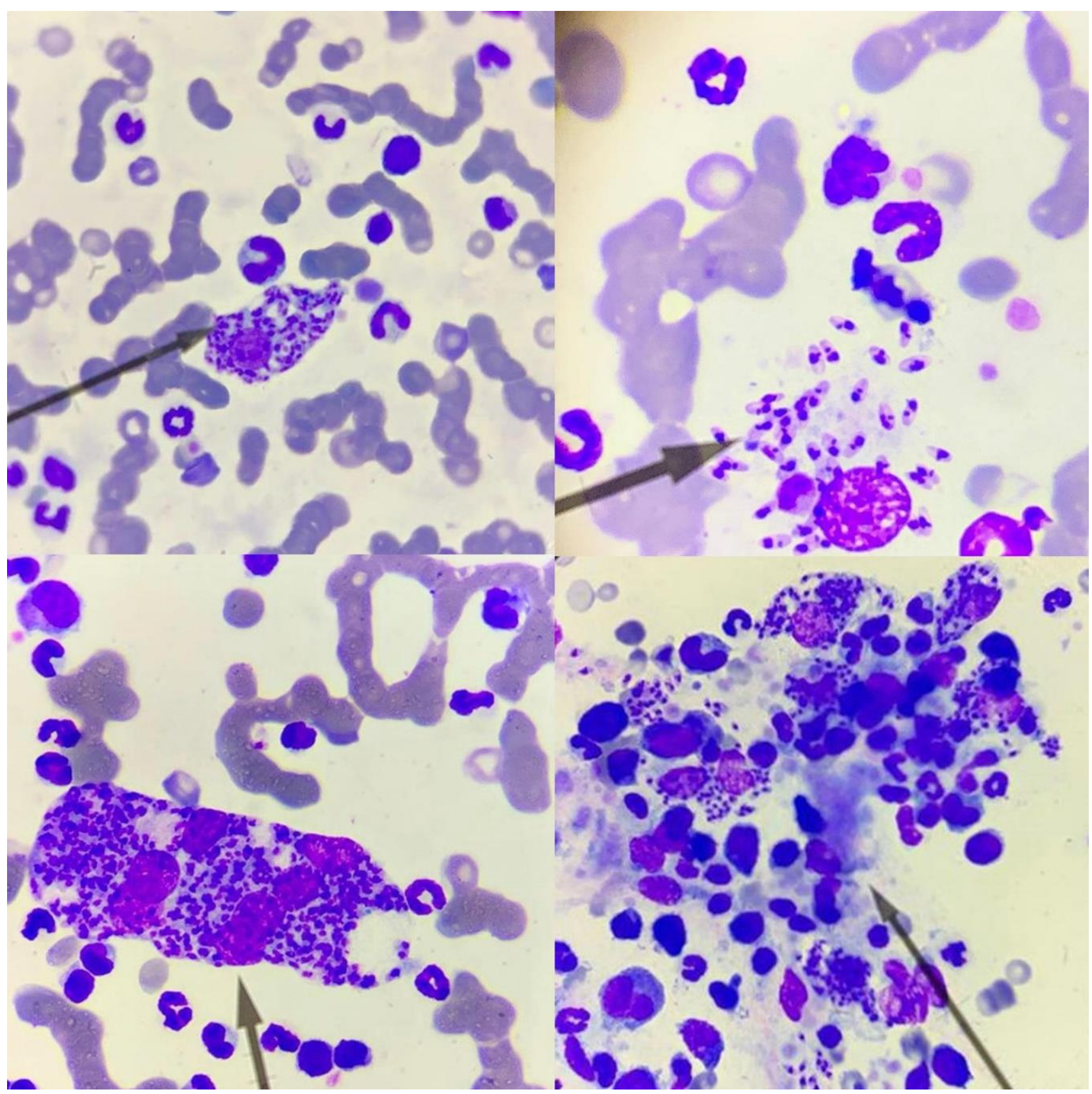

Figure 2. Bone marrow aspirate showing abundant intracellular amastigotes of Leishmania sp. (black arrows) with myeloid hyperplasia and erythroid hypoplasia, megakaryocytic hypoplasia, and plasma cell hyperplasia, as well as intracellular amastigotes, Giemsa, obtained from the English bulldog.

\section{DISCUSSION}

According to the local health authorities, and after entomological surveillance in the rural area of Pereira, no reports of Lutzomyia nor VL cases were described. In the present work, CVL was diagnosed in a dog that has never lived or stay in an endemic area. The dog had only traveled to two nearby semirural regions where humans or CVL were not informed (rural Pereira and Viterbo, rural area of Caldas, Figure 1). The presence of Lu. longipalpis or Lu. evansi has also not been documented in the Risaralda Department. However, other species of Lutzomyia transmitting cutaneous leishmaniasis species have been previously reported in Risaralda and the Coffee-Triangle Region, such as Lu. hartmanni, Lu. trapidoi, Lu. panamensis, Lu. yuilli, Lu. gomezi, Lu. columbiana, Lu. youngi, and Lu. lichyi (Bejarano et al., 2007). Differential diagnosis of spleen neoplasias or hip dysplasia should consider CVL (de Souza et al., 2005). The affecting factors may include massive migration of human and canine populations, the transition of vectors from rural areas to urban areas, the dissemination and adaptation of natural reservoirs, deforestation, climate change, and poor public health policies for the surveillance and control of this disease (Arbelaez et al., 2020). In Colombia, especially in border areas with Venezuela, forced migration from this last country has led to imported cases of leishmaniasis, including visceral forms.

The current report deserves a complete eco-epidemiological study, including searching for both canine and human VL as well as asymptomatic infections and identifying the presence of vector species in urban areas, both in Pereira and other municipalities of Risaralda, Colombia (Arbelaez et al., 2020). Moreover, VL is a life-threatening condition 
(Villamil-Gomez et al., 2019), where the World Health Organization (WHO) recommends that the animal be euthanatized or moved to areas where there are no vectors, as no effective treatment has been approved for dogs with VL (Manna et al., 2015; Dantas-Torres et al., 2019).

There have been no previous reports of urban CVL in the city of Pereira, Colombia, highlighting the need to initiate active search of human and CVL and assessing the presence of sand fly vector in the municipalities of the department of Risaralda. In this region, both human and CVL have not been documented before (Herrera et al., 2019; Arbelaez et al., 2020). The findings reinforce the establishment of surveillance and control programs in other rural and urban regions of Colombia, where favorable eco-epidemiological conditions exist (Arbelaez et al., 2020), and also the need for seroprevalence studies (Cortes, 2006). With the current report in Risaralda, in addition to Valle del Cauca, Caldas, and Putumayo (Figure 1), there are four new departments (Herrera et al., 2018) that have reported Le. infantum before non-endemic since 2018, highlighting the need for more research on VL in Colombia.

In conclusion, VL still poses a significant threat to public health, and cases as described here, signify the importance of research and identification of the circulation of Leishmania infantum/chagasi and the vectors in new areas that may become endemic and pose a risk for human transmission.

\section{DECLARATIONS}

\section{Authors' contributions}

LVGC, DKBA, MPAS, LYGH, and LCPT contributed to data and sample collection. AJRM wrote the first draft of the manuscript. LVGC, MPAS, carried out the tests and data analysis. LVGC and DKBA contributed equally for the publication of this article. All the authors critically revised the manuscript for intellectual content. All authors read and approved the final manuscript. LVGC is the guarantor of the paper.

\section{Acknowledgments}

To the San Lucas Centro Veterinario y Diagnostico, Pereira, Risaralda, Colombia for the support in reporting this case.

\section{Competing interests}

All authors declared no conflict of interests.

\section{Ethical approval}

Owners of the dog authorized and gave their written informed consent for this publication. The Institution, San Lucas Centro Veterinario y Diagnostico, where the dog was attended, acts as a warrant on the ethical monitoring of the animal care and the case report under the consent of the owners. Ethical issues (including plagiarism, consent to publish, misconduct, data fabrication and/or falsification, double publication and/or submission, and redundancy) have been checked by the authors.

\section{REFERENCES}

Arbelaez N, Moreno J, Murillo J, Montoya A, Robledo SM, Velez A and Velez ID (2020). First report of an urban case of canine visceral leishmaniasis in the municipality of cali, colombia. American Society of Tropical Medicine and Hygiene, 102(2): 289293. DOI: https://www.doi.org/10.4269/ajtmh.19-0515

Bejarano EE, Sierra D and Velez ID (2007). Dos nuevos registros de lutzomyia (diptera: Psychodidae) para el departamento de Risaralda, Colombia. Revista Colombiana de Entomología 33: 43-44. Available at : http://www.scielo.org.co/scielo.php?pid=S0120-04882007000100008\&script=sci_abstract\&tlng=pt

Cortes LA (2006). [leishmaniasis transmission focus in El Hobo, Carmen de Bolivar, Bolivar, Colombia]. Biomedica, 26, 1: $236-241$. Available at : http://www.scielo.org.co/scielo.php?script=sci_arttext\&pid=S0120-41572006000500026

Dantas-Torres F, Miro G, Baneth G, Bourdeau P, Breitschwerdt E, Capelli G, Cardoso L, Day MJ, Dobler G, Ferrer L, et al. (2019). Canine leishmaniasis control in the context of one health. Emerging Infectious Diseases, 25(12): 1-4. DOI: https://www.doi.org/10.3201/eid2512.190164

de Souza AI, Juliano RS, Gomes TS, de Araujo Diniz S, Borges AM, Tafuri WL and Santos RL (2005). Osteolytic osteomyelitis associated with visceral leishmaniasis in a dog. Veterinary Parasitology, 129(1-2): 51-54. DOI: https://www.doi.org/10.1016/j.vetpar.2005.01.008

Herrera G, Castillo A, Ayala MS, Florez C, Cantillo-Barraza O and Ramirez JD (2019). Evaluation of four rapid diagnostic tests for canine and human visceral leishmaniasis in colombia. BMC Infectious Diseases, 19(1): 747. DOI: https://www.doi.org/10.1186/s12879-019-4353-0

Herrera G, Higuera A, Patino LH, Ayala MS and Ramirez JD (2018). Description of leishmania species among dogs and humans in colombian visceral leishmaniasis outbreaks. Genetics and Evolution, 64: 135-138. DOI: https://www.doi.org/10.1016/j.meegid.2018.06.023

Manna L, Corso R, Galiero G, Cerrone A, Muzj P and Gravino AE (2015). Long-term follow-up of dogs with leishmaniosis treated with meglumine antimoniate plus allopurinol versus miltefosine plus allopurinol. Parasites \& Vectors, 8: 289. DOI: https://www.doi.org/10.1186/s13071-015-0896-0

Villamil-Gomez WE, Calderon-Gomezcaseres A and Rodriguez-Morales AJ (2019). Visceral leishmaniasis in a patient with systemic lupus erythematosus from colombia, latin america. Le Infezioni in Medicina, 27(1): 106-108. Available at : https://www.infezmed.it/media/journal/Vol_27_1_2019_18.pdf 